\title{
Respective stability of rotavirus and coronavirus in bovine milk
}

\author{
Geneviève PANON *, Sylviane TACHE **, C. LABIE ** \\ * I.N.R.A., Laboratoire de Recherches de Technologie laitière, 65, rue de Saint-Brieuc, \\ 35042 Rennes Cedex, France \\ ** Laboratoire H.I.D.A.O.A., associé I.N.R.A., Ecole Nationale Vétérinaire, \\ 23, chemin des Capelles, 31076 Toulouse Cedex, France
}

\section{Summary}

Survival or inactivation of human and animal viruses in food, water, wastes has been studied extensively.

In France, the most popular varieties of cheese, such as Camembert, Brie, Coulommiers are still prepared with raw milk, outside of heat-treated milks. These soft cheeses make up $31 \%$ of all cheeses consumed. 354000 tons were manufactured in 1985. Consumption is regularly increasing by $2 \%$ each year.

We studied the stability of 2 bovine enteroviruses: rotavirus and coronavirus, suspended in raw and sterilized bovine pre-cheese milks. The conditions of temperature, $\mathrm{pH}$, rennet action and sodium chloride concentration examined in this study are those used routinely in the manufacture of soft cheeses.

The 2 viruses have a remarquable stability to low temperatures, acid $\mathrm{pH}$, calf rennet and sodium chloride. The results are suggestive of a possible viral persistence in soft cheeses prepared with raw or heat-treated bovine milks.

Key words : Cheese - Rotavirus - Conavirus - Stability.

\section{Résumé}

Stabilité du rotavirus et du coronavirus dans le lait de vache

La survie ou l'inactivation des virus humains et animaux dans les aliments, l'eau, les effluents, a été beaucoup étudiée.

En France, les variétés les plus populaires de fromage, comme le Camembert, le Brie, le Coulommiers sont toujours préparées à partir de laits crus, en dehors de laits thermisés. Ces fromages à pâte molle correspondent à $31 \%$ de la consommation annuelle. 354000 tonnes ont été fabriquées en 1985. Cette consommation est en augmentation régulière de $2 \%$ par an.

Nous avons étudié la stabilité de deux entérovirus bovins : rotavirus et coronavirus, mis en suspension dans des laits crus et stérilisés de vache. Les conditions de 
température, $\mathrm{pH}$, action de la présure et concentration en chlorure de sodium examinées dans cette étude sont celles qui interviennent couramment dans la fabrication des fromages à pâte molle.

Les deux virus ont une remarquable stabilité à basse température, aux $\mathrm{pH}$ acides, en présence de présure et de chlorure de sodium. Les résultats suggèrent une persistance virale possible dans les fromages à pâte molle fabriqués à partir de laits crus ou thermisés de vache.

Mots clés : Fromage - Rotavirus - Coronavirus - Stabilité.

\section{Introduction}

Outbreaks of human diseases due to bacterial (Listeria, Yersinia, Campylobacter, Salmonella...) or viral (Poliomyelitis Hepatitis A, Norwalk) contaminants, suggest that water, meat, milk and dairy products, are good vehicles for the transmission of these contaminants (WaIt and SobSey, 1983; HerrmanN and Cliver, 1968 ; BarretT, 1986). Also, investigations on the persistence of Aujeszky's disease virus, Teschen virus, Swine vesicular desease virus, Bovine leukemia virus (Sullivan et al., 1971 ; Labie, 1982; Elort et al., 1986; LitTlewood et al., 1984), Foot and Mouth disease virus (Sellers, 1969 ; BlACKWELl and Hyde, 1976), in meat, ham, or milk, have been reported in the literature.

Usually, we differentiate between primary and secondary contamination of foods. Primary contamination implies that the virus is present in the raw material before harvesting the finished product. Secondary contamination (direct or indirect) takes place at any time during processing, storage or distribution until the food is eaten. It may be due to human enteric viruses. Survival or inactivation of human or animal viruses in food, milk and dairy products, waters, wastes has been extensively studied (CLIVER et al., 1970; Baumgartener et al., 1976 ; Herrmann et al., 1985; WARD et al., 1986 ; Traub et al., 1986).

Results of thermal inactivation trials of human enteroviruses demonstrated viral persistence in milk (KaPLAN and Melnick, 1954), medium (White et al., 1982), or selective organic molecules (Milo, 1971). Also, Cliver (1973) studied survival of human enteroviruses during processing of Cheddar cheese, and BLACKWELL (1976) studied survival of FMD virus in cheese.

In France, the most popular varieties of cheese are still prepared with raw milk. These soft cheeses make up $31 \%$ of consumption equivalent to 354000 $\mathrm{T}$ in 1985. Consumption is regularly increasing by $2 \%$ each year (CNIEL, 1986). We were interested in the persistence of 2 bovine enteroviruses, rotavirus and coronavirus, after experimental contamination of milk samples, acid whey fractions, and media.

We studied the survival and infectivity of our 2 bovine viruses during the different steps of cheese making. The conditions examined in this study are those used routinely in the manufacture of soft cheeses : temperature of milk during storage $\left(4^{\circ} \mathrm{C}\right)$, rennet action and curd formation at $33^{\circ} \mathrm{C}$, presence of 
low $(5 \%)$ and high $(33 \%)$ concentration of sodium chloride for brine preparation. We also studied thermal stability of both viruses at $13{ }^{\circ} \mathrm{C}, 20^{\circ} \mathrm{C}$, after heat-treatment at $63{ }^{\circ} \mathrm{C}, 72{ }^{\circ} \mathrm{C}$ and $120^{\circ} \mathrm{C}$, and in the $\mathrm{pH}$ range $4.6-6.3$.

These experimental conditions are only models to understand the influence of different parameters on the survival or inactivation of bovine enteroviruses (and probably other closely related strains), throughout cheesemaking process.

\section{Material and methods}

\section{A. Cells}

Rhesus monkey kidneys (MA 104) cells were supplied by J. COHEN, I.N.R.A., Thiverval-Grignon. Cultures were made in $75 \mathrm{~cm}^{2}$ or $150 \mathrm{~cm}^{2}$ plastic flasks. The growth medium was Eagle's minimum essential medium, EMEM, containing $10 \%$ fetal calf serum (FCS), $0.22 \mathrm{~g} / 1$ sodium bicarbonate, $0.29 \mathrm{~g} / 1$ glutamine, $100 \mathrm{U} / \mathrm{ml}$ penicillin, $100 \mu \mathrm{g} / \mathrm{ml}$ streptomycin, buffered with $20 \mathrm{mM}$ HEPES ( $N-2$ hydroxyethylpiperazine $-N^{\prime}-2-$ ethanesulfonic acid). The maintenance medium was EMEM containing $2 \%$ FCS. Monolayers were harvested with a mixture of trypsin $(0.25 \%)$ and versene $(0.5 \%)$.

Human rectal tumor (HRT 18) cells were supplied by J. LAPORTE et al. (1980). Cultures were made in $75 \mathrm{~cm}^{2}$ or $150 \mathrm{~cm}^{2}$ plastic flasks. The growth medium was RPMI 1640 , containing $15 \%$ FCS, 0.22 g/l sodium bicarbonate, $0.29 \mathrm{~g} / \mathrm{l}$ glutamine, $5 \mathrm{mg} / \mathrm{l}$ tylosine, $180 \mathrm{mg} / 1$ lincocyne, buffered with $20 \mathrm{mM}$ HEPES. The maintenance medium was RPMI 1640 supplemented by $2 \%$ FCS. Monolayers were harvested with a mixture of trypsin $(0.25 \%)$ and versene $(0.5 \%)$.

\section{B. Preparation of virus stocks}

The bovine rotavirus, strain $R F 45$, was kindly supplied by J. CoHEN. Briefly, the MA 104 monolayers were washed by MEM and incubated for $3 \mathrm{~h}$ at $37^{\circ} \mathrm{C}$. The rotavirus strain was inoculated at a low multiplicity of infection (MOI) : 0.1 plaque forming unit/cell (PFU) ; after $1 \mathrm{~h}$ adsorption, MEM containing $10 \mu \mathrm{g} / \mathrm{ml}$ trypsin, and $0.16 \%$ tryptose phosphate broth was added. After complete cytopathic effect (CPE), cells were disrupted by three cycles of freezing - thawing. The viral suspension was centrifuged at $1500 \mathrm{rpm}$ for 15 min at $4{ }^{\circ} \mathrm{C}$, then the supernatant was filtered through a $0.22 \mu \mathrm{m}$ Millipore membrane. The virus stock was stored in $1 \mathrm{ml}$ tubes, at $-20^{\circ} \mathrm{C}$. The final titer was : $4 \times 10^{9} \mathrm{PFU} / \mathrm{ml}$.

The bovine coronavirus, strain $G 110$, was kindly supplied by J. LAPORTE. The HRT 18 monolayers were washed with RPMI 1640. The coronavirus strain was inoculated at a MOI : $0.1 \mathrm{PFU} /$ cell. After $1 \mathrm{~h}$ adsorption, the maintenance medium was added to the cells. After complete CPE, cells were disrupted by one cycle of freezing - thawing. The viral suspension was centrifuged at $1500 \mathrm{rpm}$ for $15 \mathrm{~min}$ at $4{ }^{\circ} \mathrm{C}$, then the supernatant filtered through a $0.22 \mu \mathrm{m}$ Millipore membrane. The virus stock was stored in $1 \mathrm{ml}$ tubes, at $-20^{\circ} \mathrm{C}$. The final titer was $2 \times 10^{9} \mathrm{PFU} / \mathrm{ml}$. 
Milk samples: Whole raw milk was obtained from cows maintained at a university farm.

Heat-treated milks : sterilized, UHT, and pasteurized milks, were bought at a superemarket. Twenty $\mathrm{ml}$ samples were used in this study.

Whey fractions: were extracted from milks either by acid precipitation ( $\mathrm{pH} 4.6)$ or rennet coagulation ( $\mathrm{pH} \mathrm{6.3)} \mathrm{(AlAIS,} \mathrm{1984).}$

$p H: \mathrm{pH}$ of sterilized milk and media samples were ajusted to $\mathrm{pH} 4.6,5.4$ and 6.3 with $\mathrm{N} \mathrm{HCl}$. Twenty $\mathrm{ml}$ samples were contaminated by $2 \times 10^{5} \mathrm{PFU}$. Heat treatment was done for $2 \mathrm{~h}$ at $33^{\circ} \mathrm{C}$. $\mathrm{pH} 4.6$ is acid whey $\mathrm{pH}, \mathrm{pH} 5.4$ is curd $\mathrm{pH}, \mathrm{pH} 6.3$ is $\mathrm{pH}$ for rennet treatment.

$\mathrm{NaCl}$ : sterilized milk and media samples were treated with $5 \%$ and $33 \%$ $\mathrm{NaCl}$ (representative of brine solution) then contaminated by $10^{6} \mathrm{PFU} / \mathrm{ml}$. Incubation time was $7 \mathrm{~d}$ at $13^{\circ} \mathrm{C}$.

Rennet treatment: sterilized milk and media samples were treated with commercial rennet (Coopération Pharmaceutique Française, 44000 Nantes : chymosine activity $>50 \mathrm{mg} / \mathrm{l})$. Final concentration was $12.5 \mu \mathrm{g}$ for $100 \mathrm{ml}$ sample, in presence of $\mathrm{CaCl}_{2}: 0.5 \mathrm{~g} / 1, \mathrm{pH}$ 6.3. Twenty $\mathrm{ml}$ samples were contaminated with $5 \log _{10} \mathrm{PFU} / \mathrm{ml}$, then distributed in one ml aliquots. The incubation time was $7 \mathrm{~h}$ at $33{ }^{\circ} \mathrm{C}$.

Temperature action: we studied the thermal inactivation of bovine rotavirus and coronavirus suspended in raw milk, heat-treated milks and corresponding wheys. Twenty ml samples were inoculated then distributed in one $\mathrm{ml}$ aliquots. Final concentration of virus in milk and whey samples was 6.8 $\log _{10} \mathrm{PFU} / \mathrm{ml}$. Varying temperatures and times were tested : $4{ }^{\circ} \mathrm{C}$ for $13 \mathrm{~d}$, $20{ }^{\circ} \mathrm{C}$ for $10 \mathrm{~d}$. Heat treatments were done in a water-bath, at $63{ }^{\circ} \mathrm{C}$ for $1 \mathrm{~h}$ and at $72{ }^{\circ} \mathrm{C}$ for $1 \mathrm{~h}$. Contaminated samples were also heat treated at $120^{\circ} \mathrm{C}$ for $15 \mathrm{~min}$, in an autoclave.

Sample inoculations: we made direct inoculation on specific tissue culture. Before plaque assay, we treated $2 \times 1 \mathrm{ml}$ of each rotavirus sample with $10 \mu \mathrm{g} /$ $\mathrm{ml}$ trypsin solution for $1 \mathrm{~h}$ at $37^{\circ} \mathrm{C}$. All rotavirus and coronavirus samples were treated with a mixture of antibiotics : $10^{4} \mathrm{IU} / \mathrm{ml}$ penicillin, $10 \mathrm{mg} / \mathrm{ml}$ streptomycin, $2 \mu \mathrm{g} / \mathrm{ml}$ amphotericin B, and $750 \mu \mathrm{g} / \mathrm{ml}$ neomycin sulfate. The incubation was $1 \mathrm{~h}$ at $37^{\circ} \mathrm{C}$ for rotavirus samples and overnight at $4^{\circ} \mathrm{C}$ for the coronavirus samples. Antibiotic concentrations used in raw milk and whey samples provided necessary bactericidal activity.

\section{Viral plaque forming unit (PFU) assay}

\section{Rotavirus}

The protocol has been described by Scherrer et al. (1976). Confluent monolayers of MA104 cells, 4 days old, were prepared in disposable tissue culture plates (Greiner, 6 wells). After 3 washes with phosphate buffered saline (PBS), samples were inoculated onto monolayer and held for $1.5 \mathrm{~h}$ at $37^{\circ} \mathrm{C}$ in a $\mathrm{CO}_{2}$ incubator. Inoculum was removed and agarose overlay added. After 3 days, an agarose plus neutral red overlay was added. Plaques were counted after 3 to 5 days. 


\section{Coronavirus}

The protocol has been described by Vautherot (1981). Confluent monolayers of HRT 18 cells, 4 days old, were prepared in disposable tissue culture plates (Greiner, 6 wells). After 2 washes with PBS, samples were inoculated onto monolayer and held for $1.5 \mathrm{~h}$, at $37^{\circ} \mathrm{C}$ in a $\mathrm{CO}_{2}$ incubator. Inoculum was removed and agarose overlay added. After 2 days of incubation, the overlay was poured off. One $\mathrm{ml}$ of $2 \%$ rat red blood cell suspension was added, and left for $30 \mathrm{~min}$ at $37^{\circ} \mathrm{C}$. After a PBS wash, the hemadsorption plaques were counted.

\section{Results and discussion}

We first studied the rotavirus recovery from milk and the acid whey derivative inoculated with $1.5 \times 10^{4} \mathrm{PFU} / \mathrm{ml}$. Previous heat treatment of milk was pasteurization, sterilization and UHT (table 1). Approximately $93 \%$ of virus content was recovered from all heat treated milk and acid whey samples.

\section{TABLE 1}

Rotavirus recovery from milk and acid wheys samples inoculated

with $1.5 \times 10^{4} \mathrm{PFU} / \mathrm{ml}$, as a function of previous heat treatment of milks

Pourcentages de récupération du rotavirus après contamination d'échantillons de lait et de lactosérum acide en fonction du traitement thermique antérieur. Inoculum de départ $1,5 \times 10^{4} \mathrm{PFU} / \mathrm{ml}$

\begin{tabular}{l|c|c}
\hline $\begin{array}{c}\text { Milk and wheys } \\
\text { samples }\end{array}$ & $\begin{array}{c}\text { Virus recovery } \\
\text { (PFU/sample) }\end{array}$ & $\%$ \\
\hline Whole raw milk & 0 & 0 \\
acid whey & 0 & 0 \\
Whole pasteurized milk & $1.36 \times 10^{4}$ & 90 \\
acid whey & $1.44 \times 10^{4}$ & 96 \\
Whole sterilized milk & $1.36 \times 10^{4}$ & 90 \\
acid whey & $1.3 \times 10^{4}$ & 86 \\
Whole UHT milk & $1.36 \times 10^{4}$ & 90 \\
acid whey & $1.40 \times 10^{4}$ & 93 \\
\hline
\end{tabular}

TABLE 2

Coronavirus, virus recovery from milk samples inoculated with $3.3 \times 10^{4} \mathrm{PFU} / \mathrm{ml}$, as a function of previous heat treatment of milks

Pourcentages de récupération du Coronavirus après contamination d'échantillons de lait en fonction du traitement thermique antérieur. Inoculum de départ $3,3 \times 10^{4} \mathrm{PFU} / \mathrm{ml}$

\begin{tabular}{l|c|c}
\hline \multicolumn{1}{c|}{ Milk samples } & $\begin{array}{c}\text { Virus recovery } \\
\text { PFU/sample }\end{array}$ & $\%$ \\
\hline Whole raw milk & 0 & 0 \\
Whole pasteurized milk & $1.9 \times 10^{4}$ & 56 \\
Whole sterilized milk & $2 \times 10^{4}$ & 60 \\
Whole UHT milk & $1.2 \times 10^{4}$ & 36 \\
\hline
\end{tabular}


With raw milk, we observed an antirotavirus effect. The rate of inactivation was $100 \%$. Similar results were obtained in heated milk and whey samples artificially contaminated with coronavirus (table 2 ). Virus recovery was between $40 \%$ and $60 \%$ in heat treated milk samples. But the viricidal activity of whole raw milk against coronavirus was quite significant.

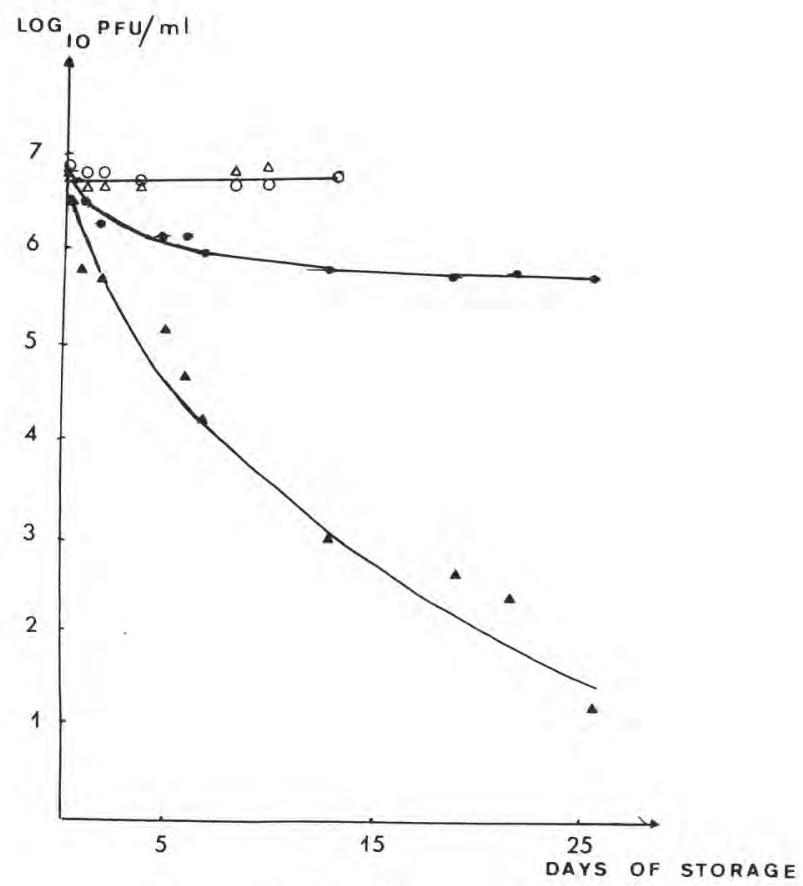

Fig. 1

Effect of storage of whey samples (sterilized bovine milk) contaminated by bovine rotavirus at $4{ }^{\circ} \mathrm{C}: \mathrm{O}-\mathrm{O} ; 20^{\circ} \mathrm{C}: \triangle-\triangle$ and bovine coronavirus at $4{ }^{\circ} \mathrm{C}:-20{ }^{\circ} \mathrm{C}$ : $\Delta-\mathbf{\Delta}$, on infectivity. $\log _{10} \mathrm{PFU} / \mathrm{ml}$.

Action de la conservation des échantillons de lactosérum (lait stérilisé de vache) contaminés par le rotavirus bovin à $4{ }^{\circ} \mathrm{C}: 0-\mathrm{O} ; 20^{\circ} \mathrm{C}: \triangle-\triangle$ et du coronavirus bovin à $4{ }^{\circ} \mathrm{C}:-120^{\circ} \mathrm{C}: \mathbf{\Delta}-\mathbf{s u r}$ leur infectivité. Log $\log _{10}$ PFU/ml.

In further experiments we studied the temporal inactivation of bovine rotavirus and coronavirus suspended in acid whey samples prepared from raw milk, and sterilized milk (tables $3,4,5$; fig. 1). Thermal inactivation studies at $4{ }^{\circ} \mathrm{C}, 20^{\circ} \mathrm{C}, 63{ }^{\circ} \mathrm{C}$, and $120^{\circ} \mathrm{C}$ confirm relative stability of bovine rotavirus in sterilized milk, the acid whey derivative or MEM, when incubated at $4{ }^{\circ} \mathrm{C}$, $20{ }^{\circ} \mathrm{C}$ for $13 \mathrm{~d}$ and $10 \mathrm{~d}$. After heat treatment at $63{ }^{\circ} \mathrm{C}$ and $72{ }^{\circ} \mathrm{C}$, rotavirus was inactivated within $20 \mathrm{~min}$ and $5 \mathrm{~min}$ respectively. Under the same experimental conditions bovine coronavirus was less stable. After incubation at $4{ }^{\circ} \mathrm{C}$ for $26 \mathrm{~d}$, and $20^{\circ} \mathrm{C}$ for $26 \mathrm{~d}$, we observed a loss of one $\log _{10} \mathrm{PFU} / \mathrm{ml}$ and $5 \log _{10} . \mathrm{PFU} / \mathrm{ml}$ respectively. After heat treatment at $63{ }^{\circ} \mathrm{C}$ and $72{ }^{\circ} \mathrm{C}$, 
coronavirus was inactivated within $5 \mathrm{~min}$ and $15 \mathrm{~s}$, respectively. After heat treatment at $120^{\circ} \mathrm{C}$, no rotavirus or coronavirus infectivity was detected after 5 $\mathrm{min}, 10 \mathrm{~min}$ and $15 \mathrm{~min}$. In the same experimental conditions, we were unable to detect viruses in raw milk samples.

TABLE 3

Rotavirus recovery from acid wheys inoculated (raw and sterilized milks) as a function of temperature and incubation time. $\log _{10} P F U / m l$. Virus input : $6.8 \log _{10} P F U / m l$

Pourcentages de récupération du rotavirus après contamination de lactosérum acide de lait cru et stérilisé, en fonction de la température et du temps d'incubation.

$\log _{10}$ PFU/ml-Inoculum de départ 6,8 $\log _{10}$ PFU/ml
(a) pas de virus
(b) non testé

\begin{tabular}{c|c|c|c|c}
\hline \multirow{2}{*}{$\begin{array}{c}\text { Days } \\
\text { of } \\
\text { storage }\end{array}$} & Raw milk & Sterilized milk & Raw milk & Sterilized milk \\
\cline { 2 - 5 } & & 6.8 & - & 6.73 \\
0 & $-(a)$ & 6.8 & - & 6.71 \\
1 & - & 6.77 & - & 6.71 \\
2 & - & 6.74 & - & 6.71 \\
3 & - & 6.71 & - & 6.71 \\
8 & - & 6.68 & ND (b) & 6.71 \\
10 & - & 6.8 & & ND \\
13 & - & &
\end{tabular}

\begin{tabular}{|c|c|c|c|c|c|}
\hline \multirow{2}{*}{$\begin{array}{l}\text { Time of } \\
\text { incubation } \\
\text { (minutes) }\end{array}$} & \multicolumn{2}{|c|}{$63^{\circ} \mathrm{C}$} & \multirow{2}{*}{$\begin{array}{c}\text { Time of } \\
\text { incubation } \\
\text { (secondes } \\
\text { and minutes) }\end{array}$} & \multicolumn{2}{|c|}{$72{ }^{\circ} \mathrm{C}$} \\
\hline & Raw milk & $\begin{array}{l}\text { Sterilized } \\
\text { milk }\end{array}$ & & Raw milk & $\begin{array}{l}\text { Sterilized } \\
\text { milk }\end{array}$ \\
\hline 0 & - (a) & 5.71 & 0 & - & ND \\
\hline 5 & $-\infty$ & 1.6 & $15 \mathrm{~s}$ & - & 6.88 \\
\hline 10 & - & 1.6 & $30 \mathrm{~s}$ & - & 6.83 \\
\hline 15 & - & 0.9 & $1 \mathrm{~min}$ & - & 6.77 \\
\hline 20 & - & - & 5 & - & - \\
\hline 30 & - & - & 15 & - & - \\
\hline 45 & - & - & 30 & - & - \\
\hline 60 & - & - & 60 & - & - \\
\hline
\end{tabular}

(a) no virus isolated.

(b) not done. 
TABLE 4

Coronavirus recovery from acid wheys inoculated (raw and sterilized milks) as a function of temperature and incubation time. $\log _{10} P F U / \mathrm{ml}$. Virus input : $6.8 \log _{10} \mathrm{PFU} / \mathrm{ml}$

Pourcentages de récupération de coronavirus après contamination de lactosérum acide de lait cru et de lait stérilisé, en fonction de la température et du temps d'incubation. $\log _{10} \mathrm{PFU} / \mathrm{ml}$ - Inoculum de départ 6,8 $\log _{10} \mathrm{PFU} / \mathrm{ml}$

(a) pas de virus

\begin{tabular}{c|c|c|c|c}
\hline \multirow{2}{*}{$\begin{array}{c}\text { Days } \\
\text { of } \\
\text { storage }\end{array}$} & Raw milk & Sterilized milk & Raw milk & Sterilized milk \\
\cline { 2 - 5 } & 0.39 & 6.87 & 2.17 & 6.87 \\
0 & $-(\mathrm{a})$ & 6.57 & - & 5.81 \\
1 & - & 6.27 & - & 5.71 \\
2 & - & 6.14 & - & 5.17 \\
5 & - & 6.07 & - & 4.64 \\
6 & - & 5.77 & - & 4.25 \\
7 & - & 5.71 & - & 2.95 \\
13 & - & 5.69 & - & 2.56 \\
19 & - & 5.70 & & 1.55 \\
22 & - & \multicolumn{2}{c}{} \\
26 & \multicolumn{2}{c}{}
\end{tabular}

\begin{tabular}{|c|c|c|c|c|c|}
\hline \multirow{2}{*}{$\begin{array}{l}\text { Time of } \\
\text { incubation } \\
\text { (minutes) }\end{array}$} & \multicolumn{2}{|c|}{$63^{\circ} \mathrm{C}$} & \multirow{2}{*}{$\begin{array}{c}\text { Time of } \\
\text { incubation } \\
\text { (secondes } \\
\text { and minutes) }\end{array}$} & \multicolumn{2}{|c|}{$72{ }^{\circ} \mathrm{C}$} \\
\hline & Raw milk & $\begin{array}{l}\text { Sterilized } \\
\text { milk }\end{array}$ & & Raw milk & $\begin{array}{l}\text { Sterilized } \\
\text { milk }\end{array}$ \\
\hline 0 & - (a) & 4.2 & 0 & - & 6.87 \\
\hline 5 & - (a) & - & $15 \mathrm{~s}$ & - & - \\
\hline 10 & - & - & $30 \mathrm{~s}$ & - & - \\
\hline 15 & - & - & $1 \mathrm{~min}$ & - & - \\
\hline 20 & - & - & 5 & - & - \\
\hline 30 & - & - & 15 & - & - \\
\hline 45 & - & - & 30 & - & - \\
\hline 60 & - & - & 60 & - & - \\
\hline
\end{tabular}

(a) no virus isolated. 
TABLE 5

Rotavirus and coronavirus recovery from acid wheys inoculated (raw and sterilized milks) after treatment at $120^{\circ} \mathrm{C}$ as a function of time. Virus input $6.8 \log _{10} \mathrm{PFU} / \mathrm{ml}$ Infectivité rédiduelle après contamination de lactosérum acide de lait cru et de lait stérilisé par le rotavirus et le coronavirus en fonction du temps de traitement à $120^{\circ} \mathrm{C}$.

Inoculum de départ $6,8 \log _{10} \mathrm{PFU} / \mathrm{ml}$

(a) pas de virus

\begin{tabular}{r|c|c|c|c}
\hline \multirow{2}{*}{$\begin{array}{c}\text { Time } \\
(\min )\end{array}$} & \multicolumn{2}{|c|}{ Rotavirus } & \multicolumn{2}{c}{ Coronavirus } \\
\cline { 2 - 5 } & Raw milk & Sterilized milk & Raw milk & Sterilized milk \\
\hline 5 & $-(\mathrm{a})$ & - & - & - \\
10 & - & - & - & - \\
15 & - & - & - & - \\
\hline
\end{tabular}

(a) no virus isolated.

\section{TABle 6}

Rotavirus and coronavirus recovery from sterilized milk and media samples at $4{ }^{\circ} \mathrm{C}$ as a function of days of storage. $\log _{10} \mathrm{PFU} / \mathrm{ml}$

Infectivité des échantillons de lait stérilisé et de milieux contaminés par le rotavirus et le coronavirus, à $4{ }^{\circ} \mathrm{C}$ en fonction des jours d'incubation. $\log _{10} \mathrm{PFU} / \mathrm{ml}$

\begin{tabular}{c|c|c|c|c}
\hline \multirow{2}{*}{$\begin{array}{c}\text { Days } \\
\text { of } \\
\text { storage }\end{array}$} & \multicolumn{2}{|c|}{ Rotavirus } & \multicolumn{2}{c}{ Coronavirus } \\
\cline { 2 - 5 } & Milk & MEM & Milk & RPMI \\
\hline $\mathrm{T}=0$ & 4.51 & 4.07 & 3.98 & 3.66 \\
$\mathrm{~T}=1$ & 4.47 & 3.94 & 3.63 & 3.23 \\
$\mathrm{~T}=2$ & ND (a) & ND & 3.47 & 2.90 \\
$\mathrm{~T}=3$ & 4.49 & 3.55 & 3.59 & 2.60 \\
$\mathrm{~T}=6$ & ND & ND & 3.68 & 2.69 \\
$\mathrm{~T}=7$ & 4.51 & 3.39 & 3.57 & 2.47 \\
\hline
\end{tabular}

(a) not done.

The thermal stability of bovine viruses was further studied in sterilized milk, MEM or RPMI 1640 media, at $4{ }^{\circ} \mathrm{C}$, for 7 days (table 6, fig. 2). Both rotavirus and coronavirus were stable in sterilized milk preparations held at $4{ }^{\circ} \mathrm{C}$. But, when suspended in medium, these viruses lost $0.6 \log _{10} \mathrm{PFU} / \mathrm{ml}$ and $1.2 \log _{10} \mathrm{PFU} / \mathrm{ml}$ of infectivity after 7 days of incubation respectively. 


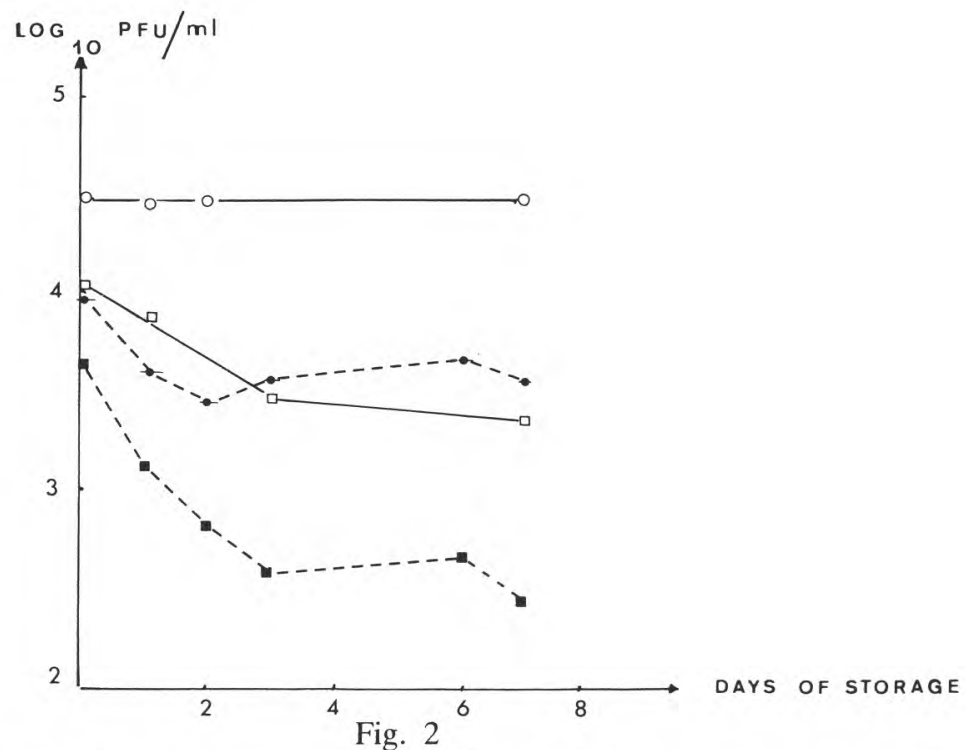

Effect of storage at $4{ }^{\circ} \mathrm{C}$ on infectivity $\left(\log _{10} \mathrm{PFU} / \mathrm{ml}\right)$ of bovine rotavirus suspended in MEM $\square-\square$, or sterilized bovine milk $\mathrm{O}-\mathrm{O}$, and of bovine coronavirus suspended in RPMI 1640 - $\mathbf{0}$, or sterilized bovine milk

Etude de l'infectivité $\left(\log _{10} P F U / m l\right)$ du rotavirus bovin suspendu en MEM $\square-\square$, ou du lait stérilisé $\mathrm{O}-\mathrm{O}$, et de celle du coronavirus bovin suspendu en RPMI $1640 \mathbf{0}-\mathbf{0}$, ou du lait stérilisé - en fonction de l'incubation à $4{ }^{\circ} \mathrm{C}$.

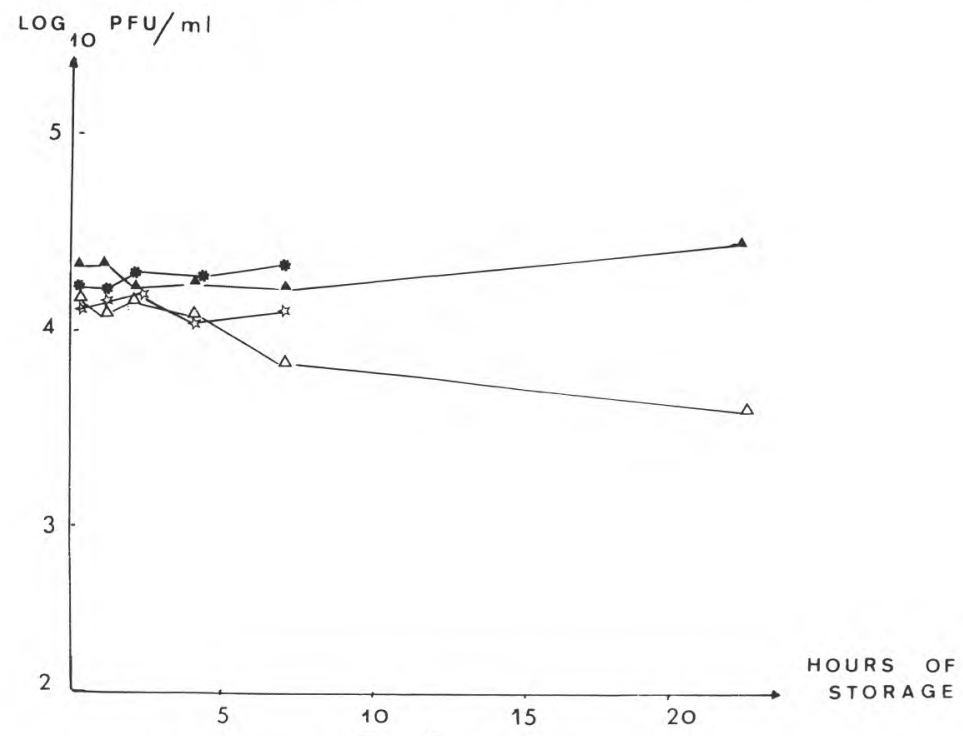

Fig. 3

Effect of incubation at $33^{\circ} \mathrm{C}$ on infectivity $\left(\log _{10} \mathrm{PFU} / \mathrm{ml}\right)$ of bovine rotavirus suspended

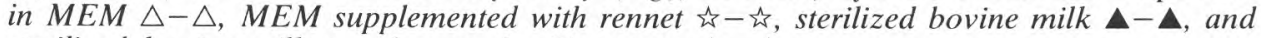
sterilized bovine milk supplemented with rennet $\star-\star$.

Effet de l'incubation à $33{ }^{\circ} \mathrm{C}$ sur l'infectivité ( $\left.\log _{10} \mathrm{PFU} / \mathrm{ml}\right)$ du rotavirus bovin suspendu en $M E M \triangle-\triangle, M E M+$ présure $\hat{\bar{s}}-\hat{z}$, en lait stérilisé $\mathbf{\Delta}-\mathbf{\Delta}$, et lait stérilisé + présure $\star-\star$. 
Bovine rotavirus infectivity was not affected after $2 \mathrm{~h}$ incubation at $33{ }^{\circ} \mathrm{C}$ at $\mathrm{pH} 4.6,5.4$, and 6.3, respectively (table 7). Rotavirus was more acid stable than bovine coronavirus. When suspended in RPMI 1640 medium, coronavirus infectivity decreased 1.4 and $0.3 \log _{10} \mathrm{PFU} / \mathrm{ml}$ respectively at $\mathrm{pH} 4.6$ and 5.4 respectively.

During $7 \mathrm{~h}$ of incubation at $33^{\circ} \mathrm{C}$, the presence of calf rennet had no specific effect on rotavirus (table 8 , fig. 3 ) and coronavirus infectivity (table 9 , fig. 4). Due to thermal inactivation, control rotavirus titer decreased $0.6 \log _{10}$ $\mathrm{PFU} / \mathrm{ml}$ when suspended in MEM, and coronavirus 2.6 and $2 \log _{10} \mathrm{PFU} / \mathrm{ml}$, respectively when suspended in RPMI 1640 medium and milk respectively. Concentration of calf rennet $(12.5 \mu \mathrm{g} / \mathrm{ml})$ sufficient for milk clotting had no effect on viral infectivity.

\section{TABLE 7}

Rotavirus and coronavirus recovery from sterilized milk and media samples as a function of $\mathrm{pH}$. Incubation $2 \mathrm{~h}$ at $37^{\circ} \mathrm{C}$. $\log _{10} \mathrm{PFU} / \mathrm{ml}$

Infectivité des échantillons de lait stérilisé et de milieux contaminés par le rotavirus et le coronavirus en fonction de $3 \mathrm{pH}$. Incubation 2 h à $37^{\circ} \mathrm{C}$. $\log _{10} \mathrm{PFU} / \mathrm{ml}$

\begin{tabular}{c|r|r|r|r}
\hline \multirow{2}{*}{$\mathrm{pH}$} & \multicolumn{2}{|c|}{ Rotavirus } & \multicolumn{2}{c}{ Coronavirus } \\
\cline { 2 - 5 } & Milk & MEM & Milk & RPMI \\
\hline \multirow{2}{*}{6.3} & 4.41 & 4.24 & 3.82 & 3.69 \\
5.4 & 4.39 & 4.25 & 3.74 & 3.34 \\
4.6 & $>4.30$ & $>4.30$ & 3.75 & 2.30 \\
\hline
\end{tabular}

TABLE 8

Kinetic of inactivation of rotavirus in sterilized milk and MEM medium, at $33{ }^{\circ} \mathrm{C}$ as a function of incubation time, and presence of rennet. $\log _{10} \mathrm{PFU} / \mathrm{ml}$

Cinétique d'inactivation du rotavirus en suspension dans du lait stérilisé et du milieu MEM à $33^{\circ} \mathrm{C}$ en fonction du temps d'incubation et de la présence de présure. $\log _{10}$ $\mathrm{PFU} / \mathrm{ml}$

\begin{tabular}{c|c|c|c|c}
\hline \multirow{2}{*}{$\begin{array}{c}\text { Hours } \\
\text { of } \\
\text { incubation }\end{array}$} & \multicolumn{2}{|c|}{$33^{\circ} \mathrm{C}$ (control) } & \multicolumn{2}{c}{$33^{\circ} \mathrm{C}+$ rennet } \\
\cline { 2 - 5 } & Milk & MEM & Milk & MEM \\
\hline $\mathrm{T}=0$ & 4.37 & 4.23 & 4.25 & 4.13 \\
$\mathrm{~T}=1$ & 4.36 & 4.11 & 4.20 & 4.17 \\
$\mathrm{~T}=2$ & 4.24 & 4.17 & 4.27 & 4.17 \\
$\mathrm{~T}=4$ & 4.27 & 4.07 & 4.27 & 4.04 \\
$\mathrm{~T}=7$ & 4.25 & 3.88 & 4.34 & $\mathrm{ND}$ \\
$\mathrm{T}=22$ & 4.32 & 3.64 & $\mathrm{ND}(\mathrm{a})$ & \\
\hline
\end{tabular}

(a) not done. 


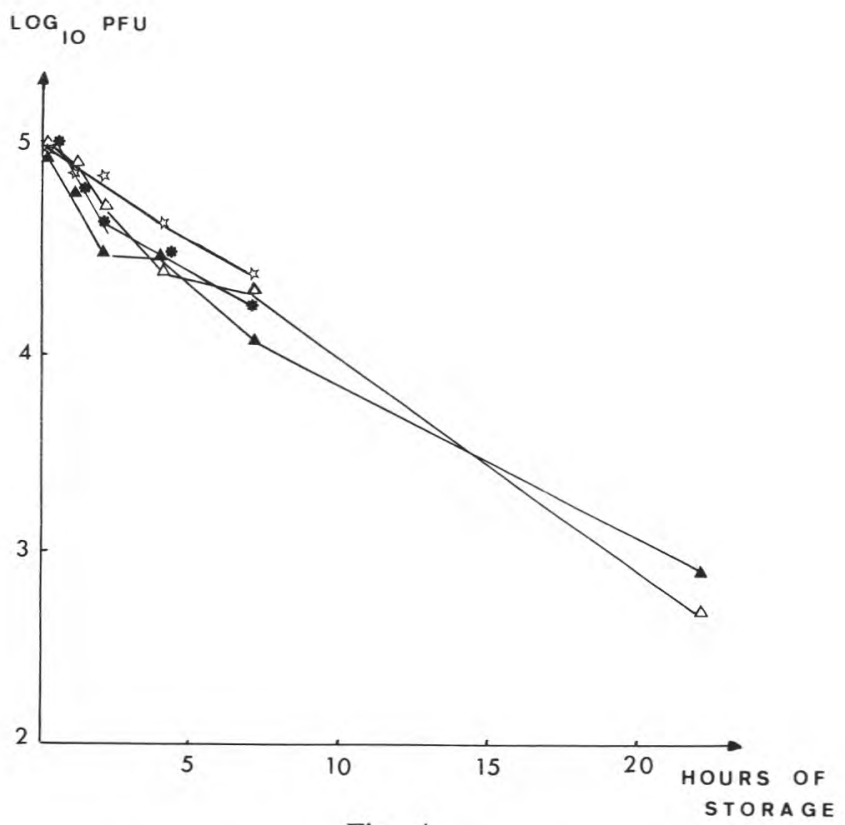

Fig. 4

Effect of incubation at $33^{\circ} \mathrm{C}$ on infectivity ( $\left.\log _{10} \mathrm{PFU} / \mathrm{ml}\right)$ of bovine coronavirus suspended in RPMI $1640 \triangle-\triangle$, RPMI 1640 supplemented with rennet $\hat{s}-\hat{s}$, in sterilized bovine milk $\mathbf{\Delta}-\mathbf{\Delta}$, and sterilized bovine milk supplemented with rennet $\star-\star$.

Effet de l'incubation à $33{ }^{\circ} \mathrm{C}$ sur l'infectivité $\left(\log _{10} \mathrm{PFU} / \mathrm{ml}\right)$ du coronavirus bovin suspendu en RPMI $1640 \triangle-\triangle$, RPMI $1640+$ présure lait stérilisé + présure $\star-\star$.

\section{TABLE 9}

Kinetic of inactivation of coronavirus in sterilized milk and RPMI 1640 medium at $33{ }^{\circ} \mathrm{C}$, as a function of incubation time and presence of rennet. $\log _{10} P F U / \mathrm{ml}$

Cinétique d'inactivation du coronavirus en suspension dans du lait stérilisé et du milieu RPMI 1640, à $33{ }^{\circ} \mathrm{C}$ en fonction du temps d'incubation et de la présence de présure. $\log _{10} \mathrm{PFU} / \mathrm{ml}$

\begin{tabular}{c|c|c|c|c}
\hline \multirow{2}{*}{$\begin{array}{c}\text { Hours } \\
\text { of } \\
\text { incubation }\end{array}$} & \multicolumn{2}{|c|}{$33^{\circ} \mathrm{C}$ (control) } & \multicolumn{2}{c}{$33^{\circ} \mathrm{C}+$ rennet } \\
\cline { 2 - 5 } & Milk & RPMI & Milk & RPMI \\
\hline $\mathrm{T}=0$ & 5. & 5.07 & 5.07 & 5.04 \\
$\mathrm{~T}=1$ & 4.82 & 4.96 & 4.82 & 4.95 \\
$\mathrm{~T}=2$ & 4.49 & 4.77 & 4.66 & 4.91 \\
$\mathrm{~T}=4$ & 4.27 & 4.47 & 4.52 & 4.74 \\
$\mathrm{~T}=7$ & 4.11 & 4.36 & 4.3 & 4.51 \\
$\mathrm{~T}=22$ & 2.93 & 2.47 & ND (a) & ND \\
\hline
\end{tabular}

(a) not done. 
TABLE 10

Kinetic of inactivation of rotavirus and coronavirus in sterilized milk and MEM or RPMI 1640 media, at $13{ }^{\circ} \mathrm{C}$, as a function of incubation time and presence of $\mathrm{NaCl}$. $\log _{10} \mathrm{PFU} / \mathrm{ml}$

Cinétique d'inactivation du rotavirus et du coronavirus en suspension dans du lait stérilisé et les milieux MEM ou RPMI 1640, à $13{ }^{\circ} \mathrm{C}$ en fonction du temps d'incubation et de la présence de $\mathrm{NaCl}$. $\log _{10} \mathrm{PFU} / \mathrm{ml}$

$13{ }^{\circ} \mathrm{C}$ (control)

\begin{tabular}{c|c|c|c|c}
\hline \multirow{2}{*}{$\begin{array}{c}\text { Days } \\
\text { of } \\
\text { storage }\end{array}$} & \multicolumn{2}{|c|}{ Rotavirus } & \multicolumn{2}{c}{ Coronavirus } \\
\cline { 2 - 5 } & Milk & MEM & Milk & RPMI \\
\hline $\mathrm{T}=0$ & 6.49 & 6.03 & 5.55 & 5.74 \\
$\mathrm{~T}=1$ & 6.41 & 6.01 & 5.25 & 5.34 \\
$\mathrm{~T}=3$ & 6.38 & 4.64 & 4.83 & 5. \\
$\mathrm{~T}=7$ & 6.50 & 4.80 & 4.41 & 4.34 \\
\hline
\end{tabular}

$13{ }^{\circ} \mathrm{C}+5 \% \mathrm{NaCl}$

\begin{tabular}{c|c|c|c|c}
\hline \multirow{2}{*}{$\begin{array}{c}\text { Days } \\
\text { of } \\
\text { storage }\end{array}$} & \multicolumn{2}{|c|}{ Rotavirus } & \multicolumn{2}{c}{ Coronavirus } \\
\cline { 2 - 5 } & Milk & MEM & Milk & RPMI \\
\hline & & 5.91 & 6.17 & 6.11 \\
$\mathrm{~T}=0$ & 6.48 & 4.85 & 6.23 & 5.97 \\
$\mathrm{~T}=1$ & 6.50 & 5.04 & 5.69 & 5.51 \\
$\mathrm{~T}=3$ & 6.55 & 4.98 & 4.84 & 4.79 \\
$\mathrm{~T}=7$ & 6.55 & &
\end{tabular}

$13{ }^{\circ} \mathrm{C}+33 \% \mathrm{NaCl}$

\begin{tabular}{c|c|c|c|c}
\hline \multirow{2}{*}{$\begin{array}{c}\text { Days } \\
\text { of } \\
\text { storage }\end{array}$} & \multicolumn{2}{|c|}{ Rotavirus } & \multicolumn{2}{c}{ Coronavirus } \\
\cline { 2 - 5 } & Milk & MEM & Milk & RPMI \\
\hline & & 4.68 & 6.04 & 4.86 \\
$\mathrm{~T}=0$ & 5.92 & 4.50 & 5.72 & 4.76 \\
$\mathrm{~T}=1$ & 6.19 & 4.38 & 5.34 & 4.38 \\
$\mathrm{~T}=3$ & 6.20 & 3.90 & 4.76 & $>2.55$ \\
$\mathrm{~T}=7$ & 6.0 & &
\end{tabular}




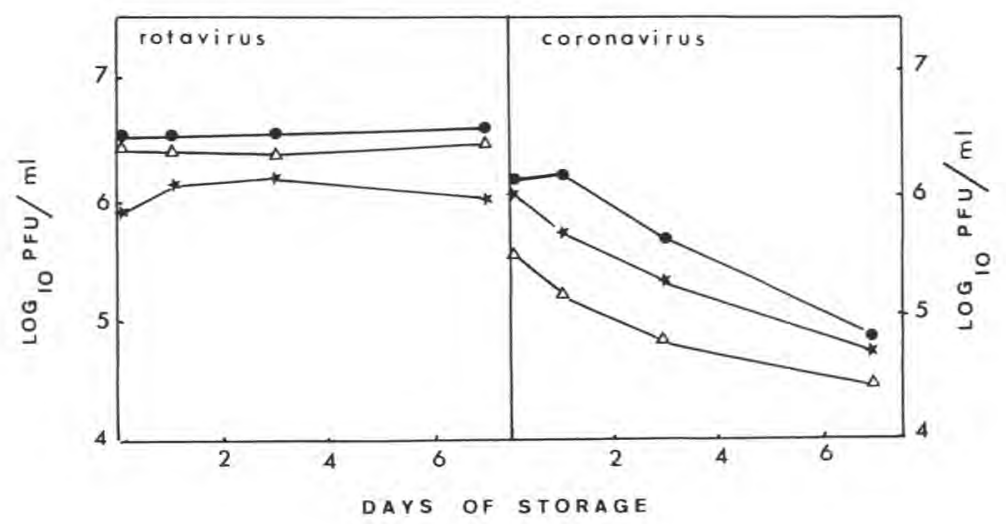

Fig. 5

Effect of $\mathrm{NaCl}$ treatment at $13{ }^{\circ} \mathrm{C}$ on the infectivity of bovine rotavirus and coronavirus suspended in sterilized bovine milk supplemented with $5 \%-1$, or $33 \% \star-\star \mathrm{NaCl}$. Control without $\mathrm{NaCl} \triangle-\triangle$.

Action du $\mathrm{NaCl}$ à $13{ }^{\circ} \mathrm{C}$ sur l'infectivité du rotavirus et coronavirus bovins en suspension dans du lait stérilisé $+5 \% \mathrm{NaCl}-\bullet$, ou $33 \% \mathrm{NaCl} \star-\star$. Contrôle sans $\mathrm{NaCl}$ $\triangle-\triangle$.

After 7 days of incubation, at $13^{\circ} \mathrm{C}$, control rotavirus suspended in MEM lost $1.2 \log _{10} \mathrm{PFU} / \mathrm{ml}$ of infectivity (table 10, fig. 5). The addition of $5 \%$ or $33 \% \mathrm{NaCl}$, had no significant virucidal effect, when virus was suspended in milk or MEM respectively. Bovine coronavirus was equally stable in milk or medium with $5 \% \mathrm{NaCl}$, and lost $1.3 \log _{10} \mathrm{PFU} / \mathrm{ml}$ of infectivity. When coronavirus was suspended in RPMI 1640 with $33 \% \mathrm{NaCl}$, the loss of infectivity was about $2.3 \log _{10} \mathrm{PFU} / \mathrm{ml}$. Rotavirus was more stable to high concentration of sodium chloride when suspended in MEM, than coronavirus.

When compared to media samples, both viruses were more stable in sterilized milk samples kept for $13 \mathrm{~d}$ and $26 \mathrm{~d}$ at $4{ }^{\circ} \mathrm{C}, 7 \mathrm{~d}$ at $13{ }^{\circ} \mathrm{C}$ or $1 \mathrm{~d}$ at $33^{\circ} \mathrm{C}$. But, after long storage of $13 \mathrm{~d}$ and $26 \mathrm{~d}$ respectively at $20^{\circ} \mathrm{C}$, bovine coronavirus lost $4 \log _{10} \mathrm{PFU} / \mathrm{ml}$ and greater than $5 \log _{10} \mathrm{PFU} / \mathrm{ml}$ of infectivity respectively.

Results concerning pasteurization treatment of bovine rotavirus and coronavirus were in agreement with our knowledge on thermal inactivation of these viruses. Other factors routinely used in the manufacture of soft cheeses, such as acid $\mathrm{pH}$, rennet and sodium chloride, have in fact no action on rotavirus infectivity, but a more significant action on coronavirus infectivity.

We observed a strong antiviral activity in raw milk. This observation was elucidated in a previous paper (PANON et al., 1987). This activity seems due to antibody like substances with neutralizing activities. It could be responsible for inapparent primary or secondary contaminations. But on the other hand, microbial and proteolytic inactivation of anteroviruses has also been studied (Cliver and Herrmann, 1972 ; Gilbert et al., 1983). 
Quality assurance for raw milk in France has to be improved (VAN DEN BERG, 1986). Since we know the relatively good stability of bovine rotavirus and coronavirus suspended in sterilized milk to factors such as low temperature, acid $\mathrm{pH}$, calf rennet or $\mathrm{NaCl}$, persistence of the two viruses throughout cheese making process could exist. Based on results of study, soft cheese prepared from raw milk could be considered as a potentially good «vehicle » for the transmission of bovine enteroviruses, and probably also for human enteroviruses which are antigenically closely related to animal strains (WoODE et al., 1976; WARD et al., 1985). Because of the demonstrated stability of these viruses in heat-treated milk, a potential exists for «secondary » contamination of these products.

Reçu le 27 mars 1987.

Accepté pour publication le 7 septembre 1987.

\section{References}

AlaIS C., 1984. Sciences du lait et principe des techniques laitières. Ed. SEPAIC, $4^{e}$ édition.

BarretT N.J., 1986. Communicable disease associated with milk and dairy products in England and Wales: 1983-1984. J. Infect., 2, 265-272.

Baumgartener L., Olson C., Onuma M, 1976. Effect of pasteurization and heat treatment on Bovine Leukemia virus. JAMA, 169, (11), 1189-1191.

Blackwell J.H., Hyde J.L., 1976. Effect of heat on Foot-and-Mouth disease virus (FMDV) in the components of milk from FMDV-Infected cows. J. Hyg. Camb., 77, 77-83.

Blackwell J.H., 1976. Survival of FMD virus in cheese. J. Dairy Sci., 59, 1574-1579.

Cliver D.O,, Kostenbader K.D., Vallenas M.R., 1970. Stability of viruses in low moisture foods. J. Milk Food Technol, 33, (11), 484-491.

Cliver D.O., Herrmann J.E., 1972. Proteolytic and microbial inactivation of enteroviruses. Water Res., 6, 797-805.

Cliver D.O., 1973. Cheddar cheese as a vehicle for viruses. I. Dairy Sci., 56, (10), 1329-1331.

C.N.I.E.L., 1986. L'économie laitière en chiffres.

Elort M., Toma B., Vuillaume A., Duret C., Parodi A., 1986. Dépistage de la leucose bovine enzootique par le test Elisa appliqué au lait de mélange : étude d'un modèle théorique. $J$. Biol. Stand., 14, 67-74.

Gilbert J.P., Wooley R.E., Shotrs E.B., Dickens J.A., 1983. Viricidal effects of Lactobacillus and yeast fermentation. Appl Environ. Micrabiol., 46, (3), 452-458.

Herrmann J.E., Cliver D.O., 1968. Methods for detecting food-borne enteroviruses. Appl. Microbiol., 16, (10), 1564-1569.

Herrmann J.E., Nowak N.A., Blacklow N.R., 1985. Detection of Norwalk virus in stools by enzyme immunoassay. J. Med. Virol., 17, 127-133.

Kaplan A.S., Melnick J.L., 1954. Effect of milk and other dairy products on the thermal inactivation of Coxsackie viruses. Am. J. Public Health, 44, 1174-1184.

LABIE C., 1982. La contamination virale des produits alimentaires d'origine animale et les risques sanitaires pouvant en résulter. Cah. Nutr. Diet., 17, (4), 197-207.

LAPORTe J., Bobulesco $P_{\text {., Rossi }} F_{\text {, }}$, 1980, Une lignée cellulaire particulièrement sensible à la réplication du Coronavirus entérique bovin. C.R. Acad. Sci. (Paris), 290 D, 623-626.

LitTlewood T.D., Tomley F.M., OWEN L.N., 1984. Preliminary report on the presence of RNAdependant DNA polymerase in canine milk. Res. Vet. Sci., 37, 97-100.

MILo G.E., 1971. Thermal inactivation of poliovirus in the presence of selective organic molecules (cholesterol, lecithin, collagen, and B-carotene). Appl. Microbiol., 21, (2), 198-202. 
Panon G., Tache S., Labie C., 1987. Antiviral substances in raw bovine milk active against bovine rotavirus and coronavirus. J. Food Prot., (under press).

Scherrer R., Cohen J., L'Haridon R., Feynerol C., Fayet J.C., 1976. Reovirus - like agent (rotavirus), associated with neonatal calf gastroenteritis in France. Ann. Rech. Vet., 7, 25-31.

Sellers R.F., 1969. Inactivation of Foot-and-Mouth disease virus in milk. Br. Vet. J., 125, 163167.

Sullivan R., Tierney J.T., Larkin E.P., Read R.B., Peeler J.T., 1971. Thermal resistance of certain oncogenic viruses suspended in milk and milk products. Appl. Microbiol., 22, (3), 315320.

Traub F., Spillmann S.K., Wyler R., 1986. Method for determining virus inactivation during sludge treatment processes. Appl. Environ. Microbiol., 52, (3), 498-503.

VAN DEN Berg M.G., 1986. Quality assurance for raw milk in the Netherlands. Neth. Milk Dairy $J ., 40,69-84$.

Vautherot J.F., 1981. Plaque assay for titration of bovine enteric coronavirus. J. Gen. Virol., 56, 451-455.

WAIT D.A., SoBSEY M.D., 1983. Method for recovery of enteric viruses from estuarine sediments with chaotropic agents. Appl. Environ. Microbiol., 46 (2), 379-385.

Ward C.W., Azad A.A., Dyall-Smith M.L., 1985. Structural homologies between RNA gene segments 10 and 11 from UK bovine, simian SA 11, and human Wa rotaviruses. Virol., 144, 328-336.

WARd R.L., KNOWLTON D.R., WinSTON P.E., 1986. Mechanism of inactivation of enteric viruses in fresh water. Appl. Environ. Microbiol., 52, (3), 450-459.

White T.C., Heidelbaugh N.D., Connel S.M., 1982. Survival of virus after thermo-processing in capillary tubes. J. Food Process. Preserv., 6, (1), 31-40.

Woode G.N., Bridger J.C., Jones J.M., Flewett T.H., Bryden A.S., Davies H.A., White G.B.B., 1976. Morphological and antigenic relationship between viruses (rotaviruses) from acute gastroenteritis of children, calves, piglets, mice and foals. Infect. Immun., 14, 804-810. 